Revista de Matemática: Teoría y Aplicaciones 4(1): 1-4 (1997)

\title{
CONVERGENCE IN NONLINEAR SYSTEMS WITH A FORCING TERM
}

\author{
J. E. NÁPOLES ${ }^{1}-$ A. I. Ruiz ${ }^{2}$
}

\begin{abstract}
The problem of convergence, as $t \longrightarrow+\infty$, of solutions of the system (1) is considered. It is assumed that the functions $\alpha, f$ and $g$ are of class $C^{1}$ for all values of their arguments, furthermore $g^{\prime}(x)>0, f^{\prime}(x) \geq r>0,0<n \leq \alpha^{\prime}(y) \leq N<+\infty$ and the functions $a(t)$ and $p(t)$ are continuous on $[0,+\infty)$ with $0<\underline{a} \leq a(t) \leq A<+\infty$ and $p(t) \geq 0$.
\end{abstract}

\section{Resumen}

Se considera el problema de la convergencia, cuando $t \longrightarrow+\infty$, de las soluciones del sistema (1). Se asume que las funciones $\alpha, f$ y $g$ son de clase $C^{1}$ para todos los valores de sus argumentos y que además $g^{\prime}(x)>0, f^{\prime}(x) \geq r>0,0<n \leq \alpha^{\prime}(y) \leq N<+\infty$ y las funciones $a(t)$ y $p(t)$ son continuas sobre $[0,+\infty)$ con $0<\underline{a} \leq a(t) \leq A<+\infty$ y $p(t) \geq 0$.

\section{Preliminars}

We consider the system:

$$
\begin{aligned}
& x^{\prime}=\alpha(y)-f(x), \\
& y^{\prime}=p(t)-a(t) g(x),
\end{aligned}
$$

where the functions involved are of class $C^{1}$ for all values of their arguments, we also assume that $g^{\prime}(x)>0, f^{\prime}(x) \geq r>0$, for all $x \in \mathbb{R}$ and $0<n \leq \alpha^{\prime}(y) \leq N<+\infty$, for all $y \in \mathbb{R}$ and the functions $a(t)$ and $p(t)$ are continuous functions on $[0,+\infty)$ with $0<\underline{a} \leq a(t) \leq A<+\infty$ and $p(t) \geq 0$.

In this paper we study the problem of convergence, when $t \longrightarrow+\infty$, of all solutions of the system (1). If the functions involved in this system are such that $\alpha(y)=y$ and $a(t) \equiv 1$, the system reduces to the well known Liénard's equation with forcing term:

$$
x^{\prime \prime}+f^{\prime}(x) x^{\prime}+g(x)=p(t) .
$$

The purpose of this note is to generalize, to the system (1), the results of [2] concerning to the convergence of all solutions of (2) to a bounded solution, under the earlier conditions.

\footnotetext{
${ }^{1}$ Department of Mathematics and Computation, isP "José de la luz y Caballero", Holguín 81000, CUBA

${ }^{2}$ Department of Mathematics, Universidad de Oriente, Santiago de Cuba 5, CP 90500, CubA
} 


\section{Results}

Let $(\varphi(t), \psi(t))$ be a bounded solution of (1). If we set $v(t)=x(t)-\varphi(t), w(t)=y(t)-\psi(t)$ we obtain the system:

$$
\begin{aligned}
v^{\prime} & =\alpha(w+\psi)-\alpha(\psi)-[f(v+\varphi)-f(\varphi)], \\
w^{\prime} & =-a(t)[g(v+\varphi)-g(\varphi)] .
\end{aligned}
$$

In the half plane $v>0$ we consider lines $w+k v=$ const., where $k \geq 0$ for $n w>a v$ and $k<0$ for $n w \leq a v$. We find conditions under which trajectories of (3) intersect these lines at points where $w=l v$, in the direction of the origin; this means that $w+k$ is decreasing on the solutions of system (3), i.e. there exist positive numbers $P$ and $Q$ such that, all the solutions of (3) cannot leave the domain $|v|<P,|w|<Q$ by crossing the boundary $v= \pm P, w= \pm Q$ when $t \rightarrow+\infty$. Differentiating $w+k v$ with (3) we obtain:

$$
k \int_{\underline{z}}^{\bar{z}} \alpha^{\prime}(r) d r-\int_{\underline{u}}^{\bar{u}}\left[a(t) g^{\prime}(s)-k f^{\prime}(s)\right] d s=v\left\{-a(t) g^{\prime}(u)-k f^{\prime}(u)+k l \alpha^{\prime}(z)\right\},
$$

where $\varphi=\underline{u} \leq u \leq \bar{u}=\varphi+v, \psi=\underline{z} \leq z \leq \bar{z}=\psi+w$. It follows from this that the conditions for intersection in the desired direction takes the form:

$$
k<\frac{a(t) g^{\prime}(u)}{l \alpha^{\prime}(z)-f^{\prime}(u)}:=R(l, t, u, z) .
$$

Let:

$$
H(l)=\inf _{t, u, z} R(l, t, u, z)
$$

we have that the infimum take place when $\alpha_{1} l>r$, for $u$ such that $f(u)<l \alpha_{1}$ and $\alpha_{1}=\inf _{z} \alpha^{\prime}(z)$. Hence we have the following:

Lemma 1 The function $R(l, t, u, z)$ reaches the infimum in the absolute extremes of the function $\alpha^{\prime}(z)$.

Let $U(l) \leq H(l)$ be any piecewise smooth function satisfying $U(l) \geq 0$ for $\alpha_{1} l>r$, $U(l)<0$ for $\alpha_{1} l \leq r$ and

$$
\lim U_{l \longrightarrow(r / N)^{-}} U(l)=-\infty .
$$

Consider the homogeneous differential equation:

$$
\frac{d w}{d v}+U(w / v)=0
$$

Let the integral curve of equation (6) which passes through the point $v=0, w=w_{0}>0$ and which lies in the right half-plane intersect the $w$-axis again at $-w_{1}<0$ (if there is no such intersection, take $\left.w_{1}=+\infty\right)$.

Theorem 1 If $w_{1}<w_{0}$, then $(x(t), y(t)) \longrightarrow(\varphi(t), \psi(t))$ as $t \longrightarrow+\infty$, where $(x(t), y(t))$ is an arbitrary solution of (1). 
Proof. Let $\gamma:(v(t), w(t))$ be a trajectory of system (1) which goes through a point $P$ of the half-plane $v>0$, by (5) and the definition of the function $U(l)$, the trajectories of the system (3) either intersect the integral curves of equation (6) in the direction of the origin or intersect the half-axis $w<0$. Assuming the opposite, suppose that the trajectory $\gamma$ lies completely inside the bounded region limited by axis $w$ and some trajectory $L_{1}$ of (6).

We write the system (3) in the form:

$$
\begin{aligned}
v^{\prime} & =-\alpha^{\prime}(\varepsilon y+(1-\varepsilon) \psi) w-f^{\prime}(\theta x+(1-\theta) \varphi) v, \\
w^{\prime} & =-a(t) g^{\prime}(\nu x+(1-\nu) \varphi) v
\end{aligned}
$$

where $0 \leq \varepsilon, \theta, \nu \leq 1 . x(t)=v(t)+\varphi(t)$ and $y(t)=w(t)+\psi(t)$ is a bounded solution, from this we have:

$$
f^{\prime}(\theta x+(1-\theta) \varphi) \leq A<+\infty, \quad g^{\prime}(\nu x+(1-\nu) \varphi) \geq \delta>0,
$$

for some positive constants $A$ and $\delta$.

Since $\alpha^{\prime}(y)$ is bounded we obtain $v^{\prime} \geq-A v+D w$ where: $D=\left\{\begin{array}{l}n \text { if } w>0 \\ N \text { if } w<0\end{array}\right.$

Then if $v(t) \geq v_{0}>0$ for all $t \geq t_{0}$ we have that $w^{\prime}(t) \leq-\delta \underline{a} v_{0}$ and $w(t) \longrightarrow-\infty$, as $t \longrightarrow+\infty$. But this is a contradiction. So:

$$
w_{1}<w_{0}
$$

From this it follows that the origin is globally stable for the system (3).

\section{Examples and related remarks}

Example 1. Let:

$$
0<\gamma \leq g^{\prime}(x) \leq \Gamma<+\infty
$$

For $U(l)$ we can take:

$$
U(l)=\left\{\begin{array}{ll}
(\underline{a} \gamma) /(n l-r) & \text { if } l>a / n \\
(A \Gamma) /(n l-r) & \text { if } l \leq a / n
\end{array},\right.
$$

when $a(t) \equiv 1$ and $\alpha(y)=y$, the condition (8) for the integral curves of equation (6) coincides with the convergence condition in (6).

Example 2. When $f^{\prime}(x) \not \equiv r$, the convergence criterion for system (1) obtained in Example 1 can be improved if, in the choice of $U(l)$, we take into account not only the greatest and least values of $g^{\prime}(x)$ and $f^{\prime}(x)$ but also relations among them. Apparently the first criterion of this type was obtained in [5]. If we consider the case where an estimate:

$$
0<\gamma \leq g^{\prime}(x) \leq s+t f^{\prime}(x)<+\infty ; \quad s, t \in \mathbb{R}_{+},
$$

holds. Then we have:

$$
U(l)= \begin{cases}(\underline{a} \gamma)(l n-r) & \text { if } l>r / n \\ ((s+t r) A)(n l-r) & \text { if } r / n>l>-s / t n \\ -t & \text { if } l \leq-s / t n\end{cases}
$$


If we set $\alpha(y)=y, a(t) \equiv 1$ and $\gamma=0$, we obtain:

$$
U(l)= \begin{cases}0 & \text { if } l>r \\ (s+t) /(l-r) & \text { if } r>l>-s / t \\ -t & \text { if } l<-s / t\end{cases}
$$

which coincides with the results given in [2].

Example 3. We take now:

$$
0<\gamma \leq g^{\prime}(x) \leq s+t \sqrt{f^{\prime}(x)}<+\infty, \quad t, s \in \mathbb{R}_{+},
$$

thus we derive that:

$$
U(l)= \begin{cases}\underline{a} \gamma /(n l-r) & \text { if } l>r / n \\ (s+t \sqrt{r}) A /(l n-r) & \text { if } r / n>l>-r / n-2 s \sqrt{r} / t n \\ t^{2} / 2\left(s-\sqrt{\left.s^{2}-t^{2} n l\right)}\right. & \text { if } l \leq-r / n-2 s \sqrt{r} / t n\end{cases}
$$

When $a(t) \equiv 1$, this result coincides with the one obtained in [8].

Example 4. We consider a forced oscillator with diferential equation:

$$
x^{\prime \prime}+c x^{\prime}+g(x)=p(t),
$$

where $c>0$ is a fixed constant, $g: \mathbb{R} \longrightarrow \mathbb{R}$ is sufficiently smooth and has limit at infinity, satisfying the inequality $g(-\infty)<g(x)<g(+\infty)$, forall $x \in \mathbb{R}$.

The function $p \in L^{\infty}(\mathbb{R})$ is bounded but not necessarily periodic. We remark that system (1) is the equation (10) under suitable conditions. [1].

Assume that $0<\gamma \leq g^{\prime}(x) \leq \Gamma<c^{2} / 4$, our Theorem 1 coincides with Theorem 1.2 in

Remark. If we take $g^{\prime}(x)$ and $f^{\prime}(x)$ bounded functions, our results are consistent with those obtained in [7].

\section{References}

[1] Alonso, J.M.; Ortega R. (1995) "Boundedness and global asymptotic stability of a forced oscillator", Nonlinear Anal. 25: 297-309.

[2] Bibikov, Y.N. (1976) "Convergence in Liénard equation with a forcing term", Vestnik LGU 7: 73-75 (russian).

[3] Nápoles, J.E. "On the boundedness and global stability of solutions of a system of differential equations ", to appear in Rev. Ciencias Matemáticas, Universidad de La Habana (spanish).

[4] Nápoles, J.E. "On the global stability of non-autonomous systems", submited for publication.

[5] Nazarov, E.A. (1977) "The coming together of the solutions of Liénard's equation" , Differencial 'nye Uravnenija 13: 1792-1795 (russian).

[6] Opial, Z. (1960-61) "Sur un théorème de C.E. Langenhop et G. Seifert", Ann. Polon. Math. 9: 145-155.

[7] Repilado, J.A.; J.E. Nápoles. "On the convergence of solutions of a bidimensional system to unique periodic solution ", submited for publication.

[8] Ruiz, A. (1993) "On the convergence of solutions of system $x^{\prime}=h(y)-f(x), y^{\prime}=-g(x)+p(t)$ to a bounded solution ", Rev. Ciencias Matemáticas, Universidad de La Habana (spanish). 